Research Article

\title{
Exploring the Possible Mechanism and Drug Targets of Huang-Qi-Gui-Zhi-Wu-Wu Decoction for the Treatment of Chemotherapy-Induced Peripheral Neuropathy on Network Pharmacology
}

\author{
Jia-lin Gu $\mathbb{D}^{1,2}$ Guo-li Wei, ${ }^{1,3}$ Yu-zhu Ma, ${ }^{1,2}$ Jin-zhi Zhang, ${ }^{1,2}$ Yi Ji, ${ }^{1,2}$ Ling-chang Li, ${ }^{1,3}$ \\ Jia-lin Yu, ${ }^{1,3}$ Can-hong $\mathrm{Hu},{ }^{1,3}$ and Jie-ge Huo $\mathbb{D}^{1,3}$ \\ ${ }^{1}$ Department of Oncology, Affiliated Hospital of Integrated Traditional Chinese and Western Medicine, \\ Nanjing University of Chinese Medicine, Nanjing, Jiangsu 210028, China \\ ${ }^{2}$ Graduate School, Nanjing University of Chinese Medicine, Nanjing, Jiangsu 210046, China \\ ${ }^{3}$ Department of Oncology, Jiangsu Province Academy of Traditional Chinese Medicine, Nanjing, Jiangsu 210028, China
}

Correspondence should be addressed to Jie-ge Huo; huojiege@jsatcm.com

Received 14 April 2020; Revised 7 November 2020; Accepted 12 November 2020; Published 23 November 2020

Academic Editor: Adolfo Andrade-Cetto

Copyright (c) 2020 Jia-lin Gu et al. This is an open access article distributed under the Creative Commons Attribution License, which permits unrestricted use, distribution, and reproduction in any medium, provided the original work is properly cited.

Chemotherapy-induced peripheral neuropathy (CIPN) is a common side effect of anticancer treatment, which may influence its successful completion. The Huang-Qi-Gui-Zhi-Wu-Wu decoction (HQGZWWD) has been widely used to treat CIPN in China although the pharmacological mechanisms involved have not been clarified. Using the network pharmacology approach, this study investigated the potential pathogenesis of CIPN and the therapeutic mechanisms exerted by the HQGZWWD herbal formula in CIPN. The targets of HQGZWWD were identified using traditional Chinese medicine (TCM) databases (TCMSP and ETCM) and prediction platforms (PharmMapper and TargetNet), and the genes of CIPN were collected by DisGeNET, GeneCards, and literature search. The common target interaction network between herbal formula and diseases was constructed by using Cytoscape. Gene Ontology (GO) function and Kyoto Encyclopedia of Genes and Genomes (KEGG) pathway enrichment analysis were used to reveal the mechanism and efficacy of HQGZWWD in the treatment of CIPN. A total of 153 CIPN-related genes were screened, and a protein-protein interaction (PPI) network with 96 nodes and 424 edges was constructed. Sixty-three active components were retrieved from HQGZWWD, with a herb-composite compound-target network including 748 nodes and 5448 edges. Forty-one targets belong to the above two networks. The analysis of network results and literature review shows that the main pathological processes of CIPN may be the inflammatory response and nerve injury, and HQGZWWD plays a therapeutic role in CIPN by regulating inflammatory response and repairing nerve injury, thus verifying the reliable efficacy of this herbal formula. In addition, we found two new potential therapeutic targets (CDK7 and GSTM2) warranting further investigation. This study fully illustrates that TCM has the characteristics of a multicompound, multitarget, and multipathway treatment, which is of great significance to study the curative effect of herbal formulations.

\section{Introduction}

Chemotherapy-induced peripheral neuropathy (CIPN) is one of the most common side effects of chemotherapy with platinum drugs, taxanes, Catharanthus roseus alkaloids, thalidomide, and bortezomib. It has been reported that the total incidence of CIPN is more than $60 \%$, while for oxaliplatin and taxanes, rates may be as high as 90\% [1]. The occurrence of CIPN is dose dependent and may be relieved by dose reduction or drug withdrawal. The emergence of CIPN seriously affects the efficacy of chemotherapy and the quality of life of patients [2]. CIPN usually occurs in the early stages of chemotherapy; its typical clinical manifestations include sensations of symmetrical burning or tingling, loss 
of sensation, and numbness at the ends of the extremities. Physical examination reveals fine motor impairment and sensory ataxia, as well as motor neuropathy and proprioceptive impairment, such as myasthenia and muscle bundle tremor $[3,4]$. Further, autonomic neuropathy such as abdominal pain, diarrhea, constipation, postural hypotension, and laryngospasm are often accompanying symptoms. Neurogenic pain is also a clinical symptom, which is more likely to have more of a physical and mental impact on patients, and often needs auxiliary pain relief and antidepressant therapy [5]. The emergence of CIPN will lead to tension in patients, affect their quality of life, reduce the treatment dose, prolong chemotherapy treatment, or even stop treatment, thus interfering with the achievement of the desired effect.

The management of CIPN has entered a stage of comprehensive treatment. Traditional Chinese Medicine (TCM), as an important part of the Complementary and Alternative Medicine (CAM) approach, plays a key role. Increasing data show that TCM has an obvious efficacy in the prevention and treatment of cancer and its complications, including radiotherapy, chemotherapy, and postoperative recovery [6-8].

The Huang-Qi-Gui-Zhi-Wu-Wu decoction (HQGZWWD) was first published in the Synopsis of Golden Chamber written by Zhang Zhongjing, a medical scientist in the Han Dynasty (25 AD), and represents one of the more commonly used clinical herbal formulations. It consists of five herbs: Hedysarum Multijugum Maxim (Huang Qi), Cinnamomi Ramulus (Gui Zhi), Paeoniae Radix Alba (Bai Shao), Zingiber Officinale Roscoe (Sheng Jiang), and Jujubae Fructus (Da Zao). Its main function is to supplement qi, the warming meridian, and to ease pain. HQGZWWD is mainly used for the hand-foot syndrome, CIPN, diabetic peripheral neuropathy, and rheumatoid arthritis. Previously, we have confirmed the efficacy of HQGZWWD in the prevention and treatment of CIPN without reducing antitumor activity of chemotherapeutic drugs in the animal model and cancer patients [9]. However, the potential therapeutic mechanism involved has not been fully elucidated.

Network pharmacology is based on the similarity of the structure and efficacy of drugs to construct a drug-target network to explore the mechanisms of action of different agents. It emphasizes the multichannel regulation of signaling pathways to improve the therapeutic effects of drugs and to reduce their toxicity and adverse effects, so as to improve the success rate of new drug clinical trials and save on the costs involved in drug research. Network pharmacology is a current research hotspot applied to clarify the effective components and treatment activity of TCM [10]. It has been widely used to explore the therapeutic mechanisms of TCM in diseases involving the cardiovascular and nervous systems, respiratory diseases, diabetes, cancer, and osteoarthropathy and has achieved significant results [11-16]. The purpose of this study was to analyze the pathogenesis of CIPN and the potential therapeutic mechanisms of HQGZWWD through network pharmacology, so as to provide a theoretical basis for the clinical application of herbal formulations.

\section{Methods of Data Preparation}

2.1. Composite Compounds of HQGZWWD. ADME refers to the process of absorption, distribution, metabolism, and excretion of exogenous compounds, which can reflect the dynamic changes of drug activity in animals or humans. It is an important guide to the development of new drugs and to the design of compounds [17]. We searched the Traditional Chinese Medicine Systems Pharmacology Database and Analysis Platform (TCMSP, http://tcmspw.com/tcmsp.php), which is the most widely used information query platform for TCM, to identify the composite compounds and targets of HQGZWWD [18]. A total of 790 compounds were identified: 87 in Hedysarum Multijugum Maxim, 220 in Cinnamomi Ramulus, 85 in Paeoniae Radix Alba, 265 in Zingiber Officinale Roscoe, and 133 in Jujubae Fructus. All molecules were screened according to the ADME criteria (oral bioavailability $>30 \%$ and drug likeness $>0.18$ ) recommended by TCMSP $[19,20]$. A total of 63 compounds were selected. Notably, 14 of these effective compounds were verified by ultraperformance liquid chromatographyquadrupole-time-of-flight mass spectrometry (UPLC-QTOF-MS) technology in our previous study, and several compounds are included: jaranol, isorhamnetin, formononetin, calycosin, kaempferol, (3R)-3-(2-hydroxy-3,4dimethoxyphenyl)chroman-7-ol, quercetin, sitosterol, (+)-catechin, paeoniflorin, benzoyl paeoniflorin, and poriferast-5-en-3beta-ol (Supplementary Tables S1 and S2).

2.2. Compound Targets of HQGZWWD. In order to make the targets of HQGZWWD more comprehensive, we have added descriptions of target information from the Encyclopedia of Traditional Chinese Medicine (ETCM, http:// www.nrc.ac.cn:9090/ETCM/). The ETCM was updated in 2018 and provides a more professional ADME evaluation and enhanced information on compounds and targets [21]. Based on the limitations of the database, we also used PharmMapper (http://lilab-ecust.cn/pharmmapper/) and TargetNet (http://targetnet.scbdd.com/), both of which are target prediction platforms. The former predicts the target using a pharmacophore model, which models the molecular docking of compounds through characteristic features and the spatial arrangement of pharmacologically active molecules [22]. TargetNet builds a large number of quantitative structure activity relationship (QSAR) models based on the input compounds to predict targets [23]. Next, UniProt (https://www.uniprot.org/) was used to standardize all target names. Some compounds for which the target information could not be identified were excluded. In total, $48 \mathrm{com}$ pounds were selected (Supplementary Table S3).

\subsection{Targets of CIPN}

2.3.1. Targets from Known Databases. The molecular targets for CIPN were obtained from GeneCard (https://www. genecards.org/) and DisGeNET (https://www.disgenet.org/). GeneCard is an authoritative platform for human gene annotation information that integrates human gene information 
from other databases [24]. DisGeNET integrates a large number of data about disease-associated genes and variants from multiple sources, covering almost all areas of human diseases [25]. In addition, we searched the PubMed database (https://www.ncbi.nlm.nih.gov/pubmed/) to supplement information on the disease gene.

2.3.2. Target Supplement. GeneMANIA (http://genemania. $\operatorname{org} /$ ), developed by the University of Toronto, was used to analyze and identify dominant genes and provided genomic and proteomic data to discover functionally similar genes [26]. We uploaded the disease targets downloaded from GeneCard and DisGeNET to the database and output a list of 20 resultant genes.

Through database screening, literature search, and prediction target supplements, a total of 153 disease genes were selected (Supplementary Table S4).

2.4. Protein-Protein Interaction Network. The protein-protein interaction (PPI) networks in this article were all derived from String V11.0 (https://string-db.org/), which was updated in November 2018, to search for functional interactions between proteins and help to mine core regulatory genes in the network [27].

2.5. Network Construction. We constructed three networks: (i) the CIPN network, (ii) the herbal-compounds-targets network for HQGZWWD, and (iii) the HQGZWWD-CIPN overlapping targets network. All networks were built through the network visualization software Cytoscape (V3.7.2 https://cytoscape.org/), an open source network that focuses on data visualization and analysis. Its core function is to provide a basic functional visual layout and query network based on the combination of basic data [28]. In addition, we modularized the CIPN network through the Mcode cluster function in Cytoscape, which is conducive to better mining the core functions of the network.

2.6. Gene Ontology Functional and Kyoto Encyclopedia of Genes and Genomes Pathway Enrichment Analysis. The Gene Ontology (GO) function and Kyoto Encyclopedia of Genes and Genomes (KEGG) pathway enrichment analyses of all targets were obtained from the Database for Annotation, Visualization and Integrated Discovery (DAVID, V6.8, https://david.ncifcrf.gov/), which integrates biological data and analysis tools to provide comprehensive biological function annotation information for the large-scale gene or protein lists [29].

\section{Results}

3.1. The CIPN Network. Information relative to 150 targets was extracted from String, and 96 nodes and 424 edges were constructed under the condition of high confidence. In this network, targets with a higher degree are regarded as the core targets (32 in STAT3, 31 in IL6, 28 in TNF, 24 in IL10, 24 in VEGFA, 23 in TP53, 22 in IL2, 22 in IL4, and 21 in
CSF2). This suggests that these genes may be the key genes or central genes affecting the development of CIPN (Figure 1).

3.2. GO Functional Analysis of the CIPN Network. Through the modular analysis of the CIPN network using the Mcode cluster, the entire network was divided into three clusters (Figure 2).

These clusters were then interpreted using GO biological process (GO-BP) analysis. The top 10 most significantly enriched BP terms in each group were selected for analysis. The results showed that cluster 1 included the immune response, cellular response to lipopolysaccharide, positive regulation of nitric oxide biosynthesis, positive regulation of chemokine biosynthesis, and inflammatory response. Cluster 2 included mechanisms involving transcriptioncoupled nucleotide-excision repair, transcription elongation from the RNA polymerase I promoter, nucleotide-excision repair, and DNA incision. Cluster 3 included processes involved in glutathione derivative biosynthesis, cellular detoxification of nitrogen compounds, glutathione metabolism, and xenobiotic metabolism (Figure 3).

3.3. KEGG Pathway Enrichment Analysis of CIPN Targets. All disease genes were uploaded to DAVID, and 13 pathways were obtained, of which four pathways contained more enriched genes (i.e., 20 in pathways in cancer, 18 in the PI3K-Akt signaling pathway, 13 in the Jak-STAT signaling pathway, and 10 in the MAPK signaling pathway) (Figure 4).

3.4. Herbal-Compounds-Targets Network of HQGZWWD. This network consisted of 728 nodes ( 5 herbal nodes, 48 compound nodes, and 675 compound-target nodes) and 5448 edges, of which 6 compounds (mairin, beta-sitosterol, $(+)$-catechin, quercetin, stigmasterol, and kaempferol) appeared in more than two herbs, and many targets (such as GSTM1, GSTM2, ACHE, and ESR2) that were targets of multiple compounds. In addition, 41 overlapping targets (peripheral nodes, such as CYP2C8, GSTM1, AGXT, GSK3B, and ABCC4) were identified after comparing herbal formula targets with CIPN targets, of which three targets (AGXT, ABCC4, and PPARD) were not identified in the previously constructed disease network, and two targets (CDK7 and GSTM2) were obtained from the predictive platform. Finally, using the DAVID platform, we found that the targets in herbal formulations were enriched in a variety of diseases such as lung cancer, type 2 diabetes, bladder cancer, chronic obstructive pulmonary disease, colorectal cancer, and chronic renal failure (Figure 5).

3.5. HQGZWWD-CIPN Overlapping Targets Network. The above 41 overlapping targets were inserted into the PPI network and denominated the HQGZWWD-CIPN common target network. A total of 40 nodes and 247 edges were constructed, in which 7 targets had a higher degree in the network (29 in TP53, 27 in ALB, 23 in IL6, 23 in VEGFA, 22 in CASP3, 21 in TNF, and 20 in CYP3A4). These nodes were 


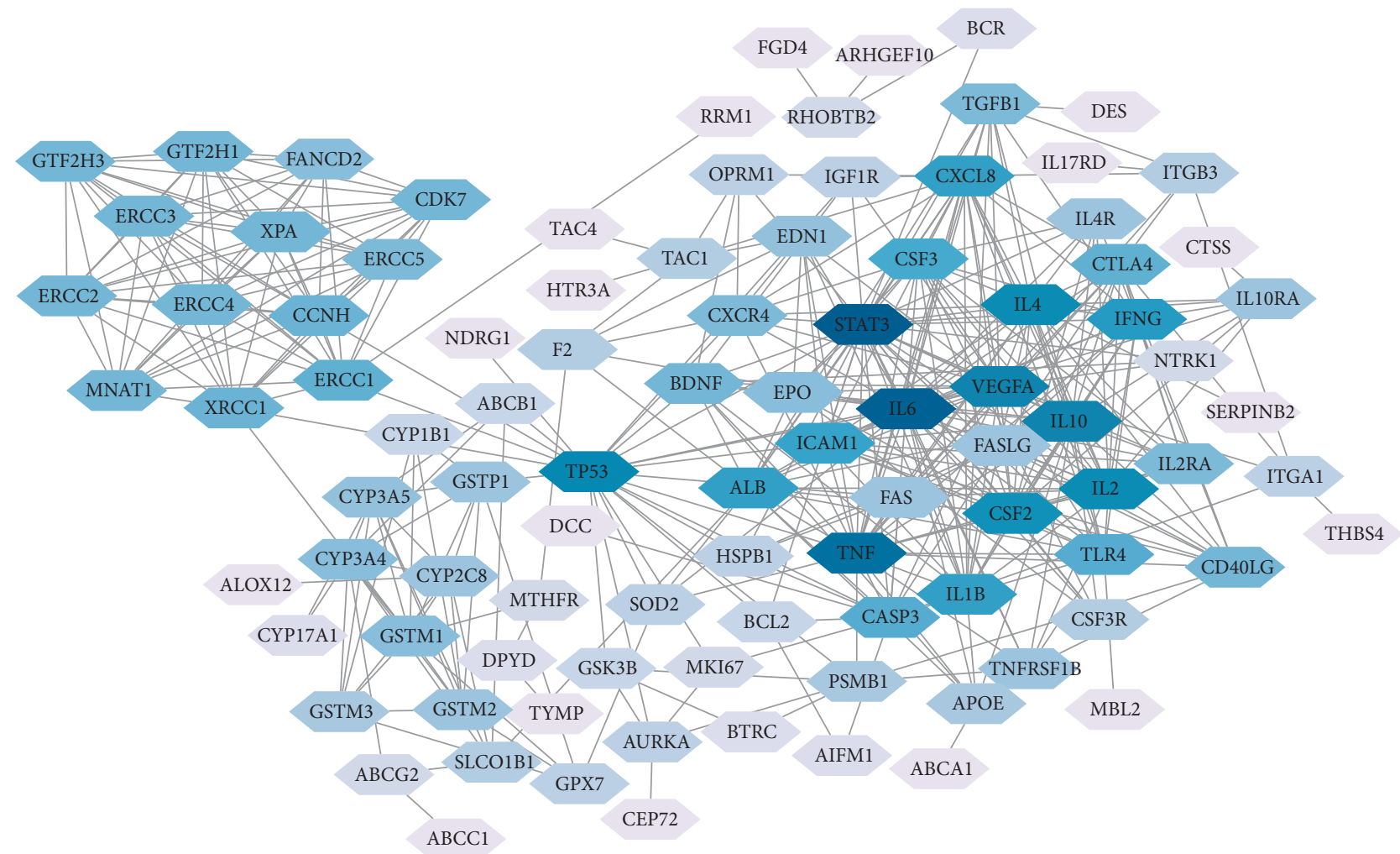

FIGURE 1: CIPN target protein-protein interaction network. Nodes with a darker color have a higher degree in the network.

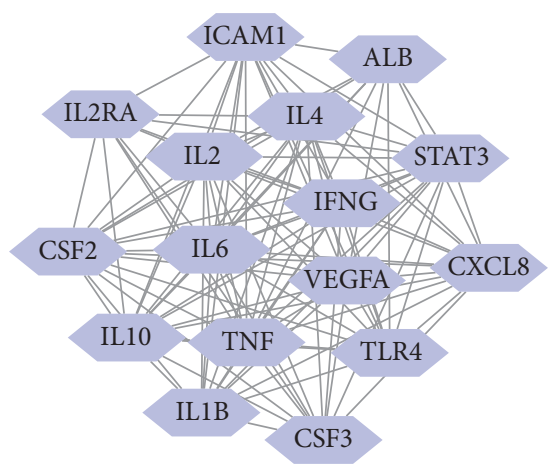

(a)

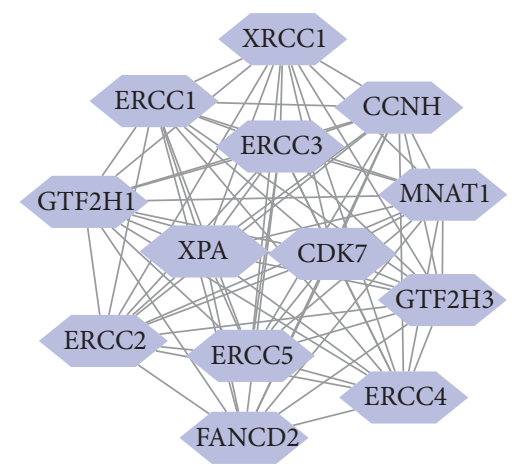

(b)

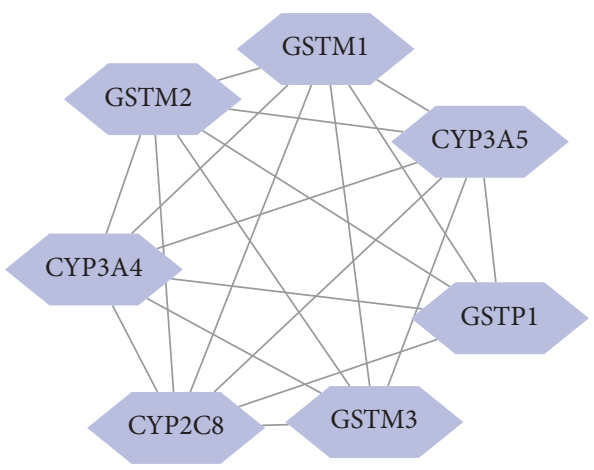

(c)

FIGURE 2: Mcode clustering of CIPN targets in the protein-protein interaction network. (a-c) represent clusters 1, 2, and 3, respectively.

predicted to play a core role in the treatment of CIPN with HQGZWWD (Figure 6).

3.6. GO Functional and KEGG Pathway Enrichment Analysis of Overlapping Targets. To study the biological functions and metabolic pathways of these key targets, DAVID analysis was employed to analyze overlapping targets. GO functional analysis revealed the following target-associated terms: (i) "biological processes (BP)" involving negative regulation of apoptosis mechanisms, immune responses, positive regulation of chemokine biosynthetic processes, and positive regulation of nitric oxide biosynthetic processes; (ii) "cell components (CC)" comprising the extracellular space, external side of the plasma membrane, and extracellular region; and (iii) "molecular functions (MF)" involving cytokine activity, oxygen binding, growth factor activity, and glutathione binding. KEGG pathway enrichment analysis showed that the overlapping targets were mainly involved in cytokine and inflammatory responses as well as the T-cell receptor, NF-kappa B, HIF-1, TNF, and Jak-STAT signaling pathways (Figures $7(\mathrm{a})$ and $7(\mathrm{~b})$ ).

\section{Discussion}

At present, the mechanisms involved in CIPN have not been fully elucidated and may include disruption of neuronal axonal transport, mitochondrial dysfunction, inflammatory stimulation, oxidative stress, nerve injury, and changes in ion channel activity. The 


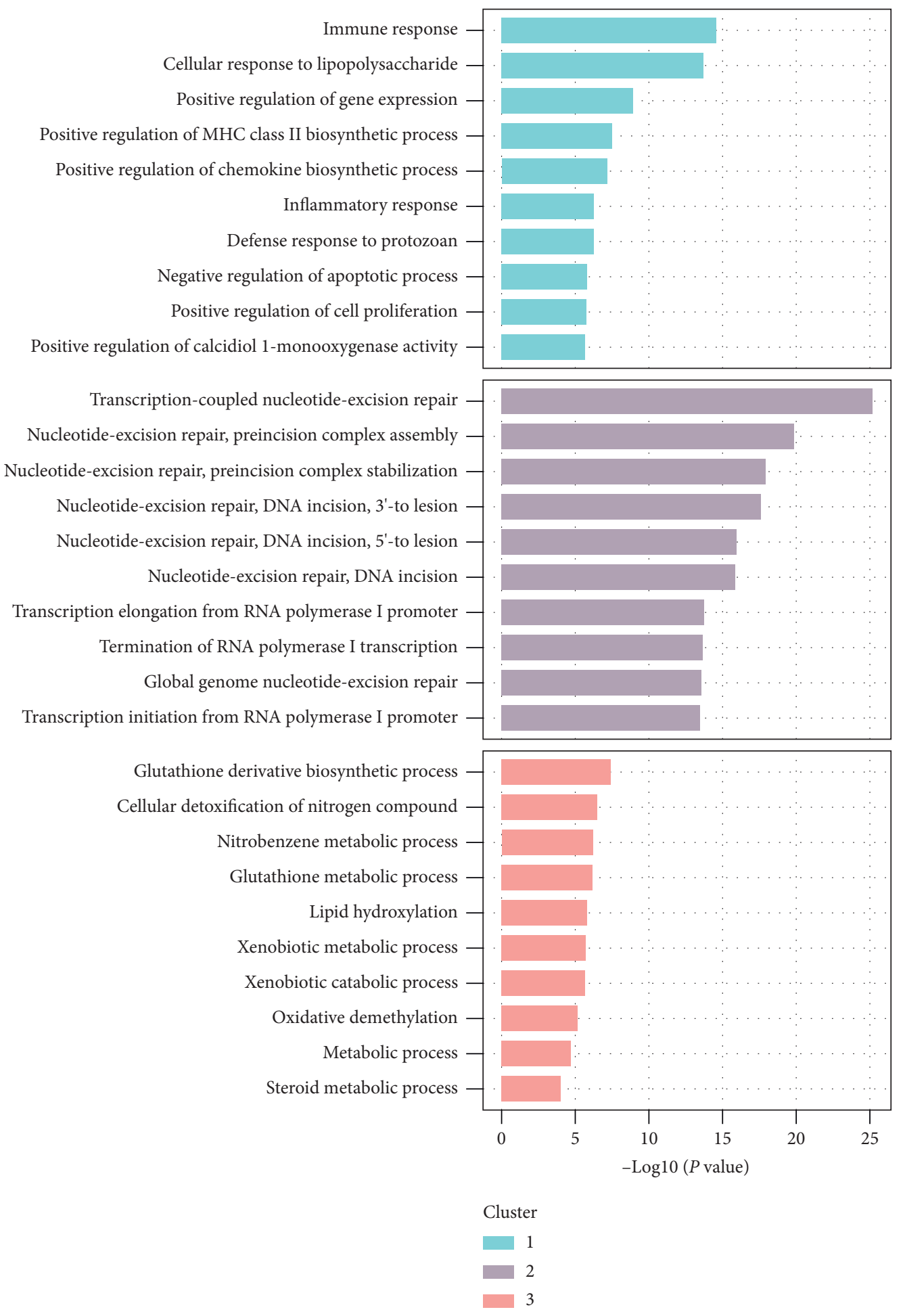

FIgURE 3: GO biological process analysis of CIPN clusters.

pathogenesis of CIPN is mainly associated with injury to sensory neurons in the dorsal root ganglion caused by chemotherapeutic drugs. The incidence and severity are not only related to individual factors but also include the type of agent, cumulative dose, treatment schedule, and treatment time [30]. The classic treatment strategy is still prevention and management of symptoms [31]. Ion channel modulators, neuroprotective agents, antioxidants, tricyclic antidepressants, and antiepileptic agents are commonly used in clinical treatment $[32,33]$. The only agent currently recommended for the treatment of neuralgia caused by CIPN is duloxetine [34, 35]. A "stop-and-go" strategy is generally adopted, which involves stopping treatment with the drug immediately, reducing the drug dose, or prolonging the time of chemotherapy and then resuming treatment after the symptoms are relieved [36]. 


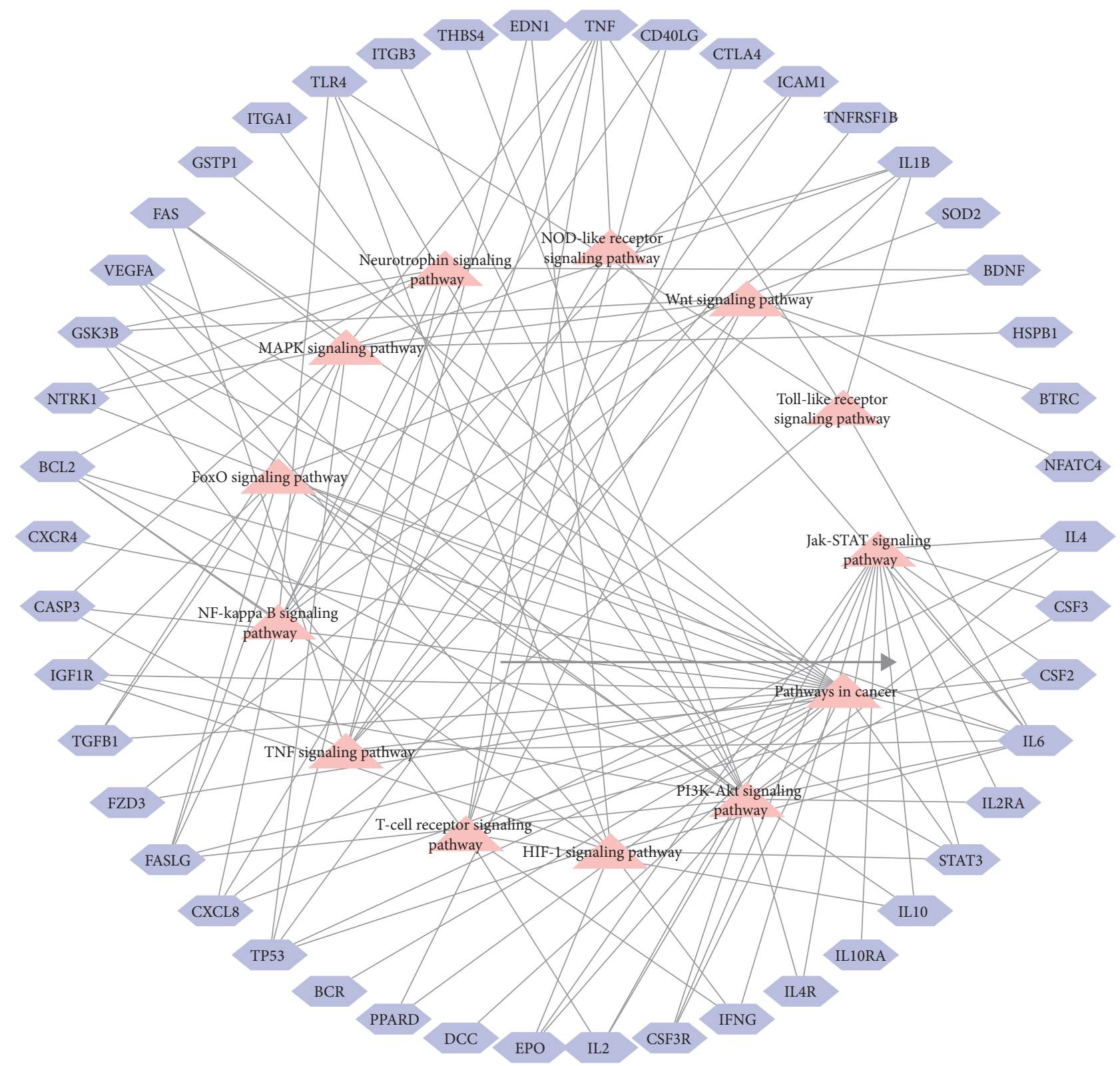

FIgURE 4: KEGG pathways of CIPN targets.

Through previous animal studies, we determined that HQGZWWD could reduce the intake of platinum in the dorsal root ganglion of the oxaliplatin rat model and could promote platinum pumping, so as to reduce accumulation of platinum and prevent chronic peripheral neurotoxicity induced by exposure to oxaliplatin [37]. A meta-analysis showed that HQGZWWD could effectively prevent and reduce oxaliplatin-related peripheral neurotoxicity [38]. Therefore, we propose that HQGZWWD can effectively reduce and alleviate the incidence and severity of CIPN. Through network pharmacology, we constructed the core networks involved in CIPN and mechanisms of action of herbal formulations to explore the underlying pathogenesis of CIPN and to explore any molecular therapeutic mechanisms and/or pathways potentially associated with HQGZWWD.
First, we constructed and analyzed the core network of CIPN, in which regulatory genes including STAT3, IL6, TNF, IL10, VEGFA, TP53, IL2, IL4, and CSF2 were identified. Among these was STAT3, which not only participates in the signal transduction pathways of many cytokines including interferon, interleukins, and growth factors but also regulates important functional activities such as cell growth, differentiation, migration, apoptosis, autophagy, immunity, and metabolism. Studies have shown that STAT3 is not only involved in the inflammatory response but also mediates tumorigenesis and stages of carcinogenesis [39, 40]. Furthermore, STAT3 also has an effect on peripheral nerve cell regeneration and participates in nerve repair [41]. The cytokines IL6, TNF, IL10, IL2, and IL4 are all immunomodulatory factors, among which TNF and IL-6 have been confirmed to be involved in the pathological process of 


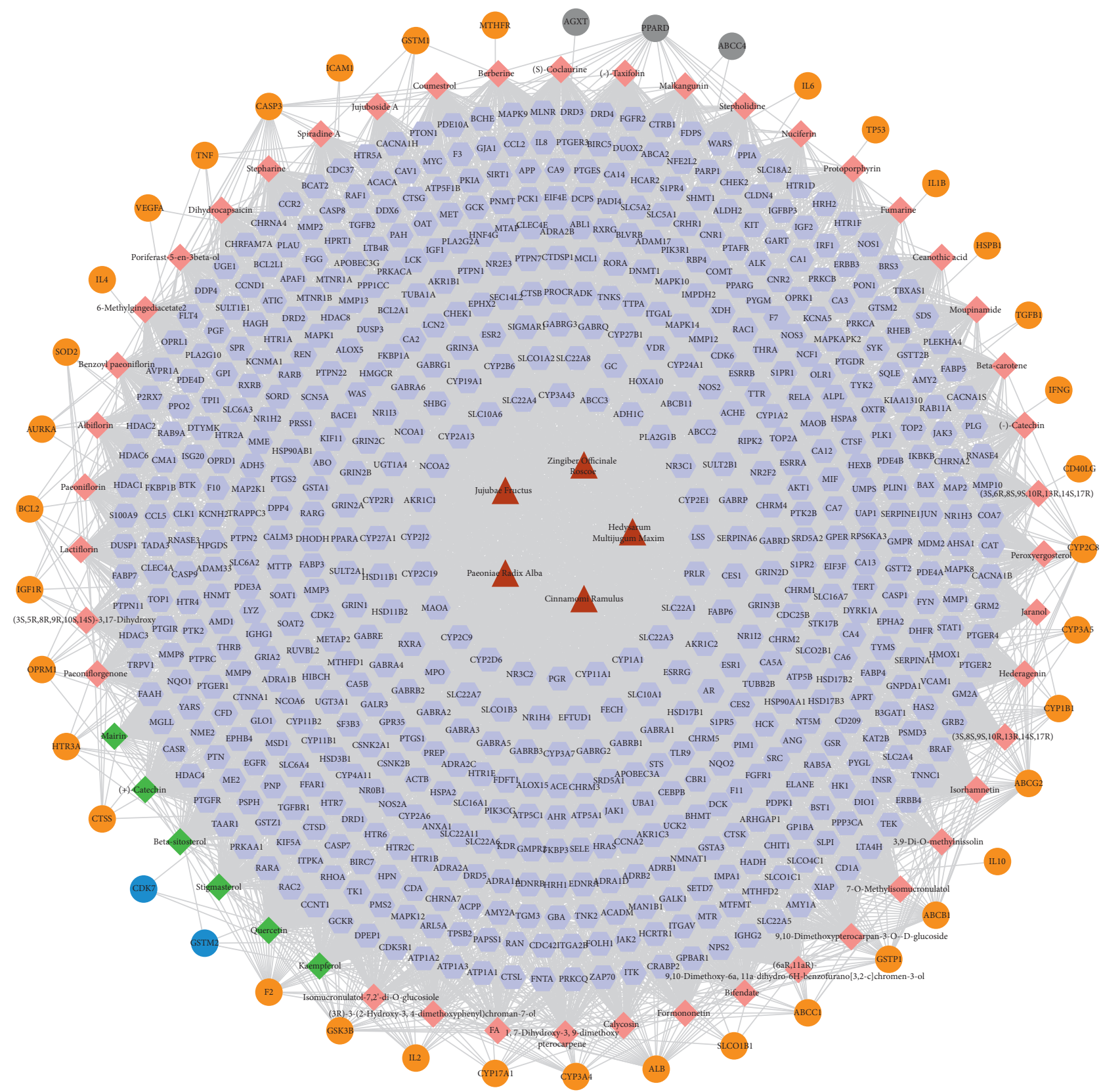

FIGURE 5: Herbs-compounds-targets network of HQGZWWD. Red triangle nodes represent herbs, diamond nodes represent compounds, green diamond nodes represent repeated compounds in herbs, peripheral nodes represent overlapping targets between HQGZWWD and CIPN, gray round nodes were not identified in the previously constructed CIPN targets network, and blue rounds represent targets derived from predictive data.

peripheral nerve injury $[42,43]$. The core biological processes of CIPN include the immune response, positive regulation of nitric oxide biosynthesis, inflammatory response, and positive regulation of chemokine biosynthesis. Nitric oxide exerts both anti-inflammatory and proinflammatory regulatory effects. Its proinflammatory activity is mainly manifested in the promotion of the proliferation of inflammatory cells and tissue injury. An imbalance in nitric oxide levels is the key factor causing neuropathic pain [44]. Through pathway enrichment analysis, we found that most genes we identified were enriched in inflammation-related pathways, including the Jak-STAT, NF-kappa B, MAPK, and Toll-like receptor signaling pathways. The Jak-STAT signaling pathway is widely involved in cell proliferation, differentiation, and apoptosis, which can promote the occurrence and development of inflammation and tumors. Dominguez et al. [45] found that this pathway can be activated in the spinal cord microglia of rats with peripheral nerve injury and leads to neuropathic pain. Essentially, we found that the main pathological processes of CIPN may involve the inflammatory response and nerve injury, and our results also indirectly explain the complexity of CIPN 


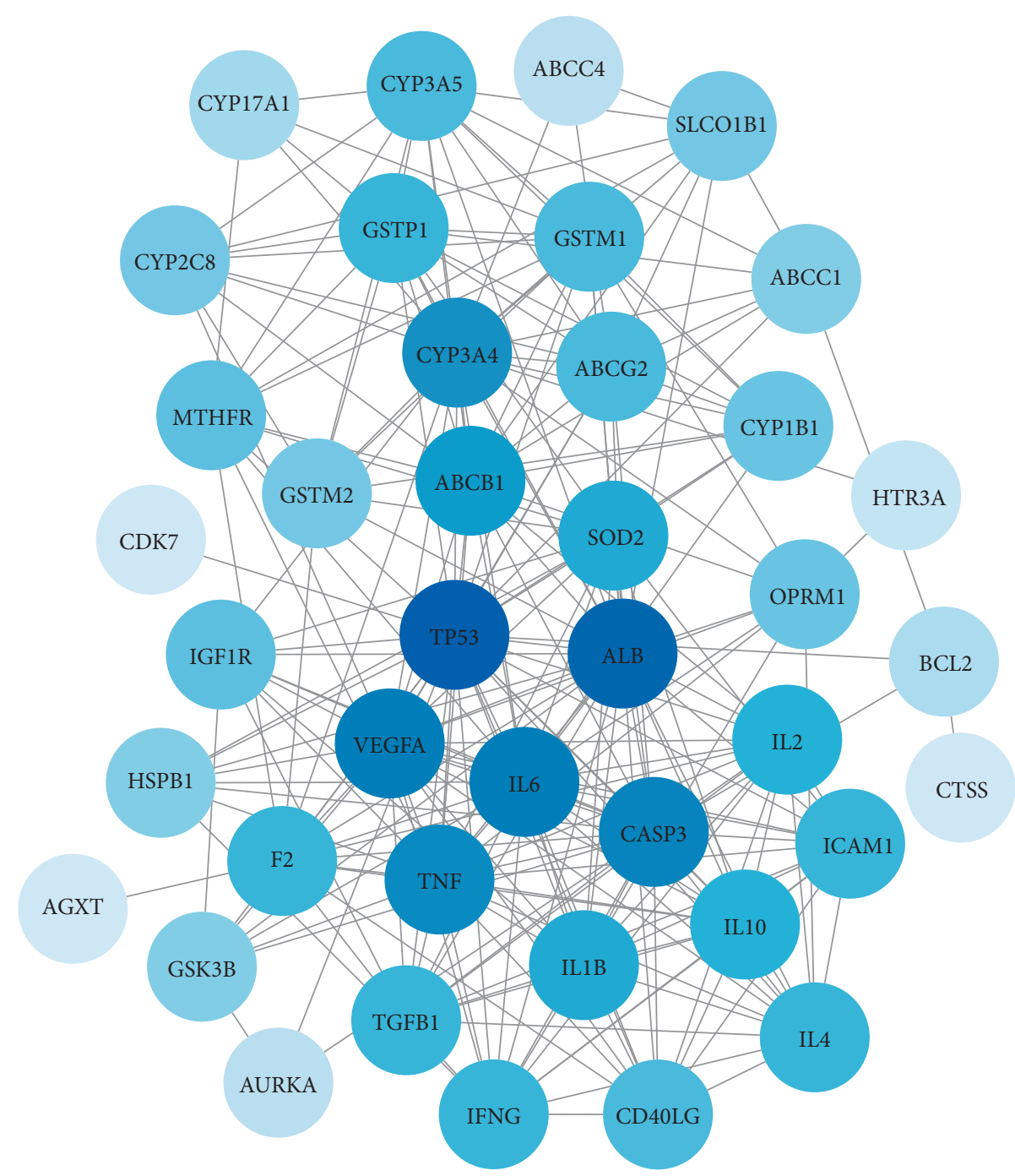

FIgURE 6: Overlapping targets network. The node having a darker color has a higher degree in the network.

pathogenesis, and these key pathways provide a direction for the future development of new drugs.

Secondly, we explored the herbal compounds and their targets. Our results showed that HQGZWWD comprises a variety of active compounds. For example, quercetin is a flavonoid widely found in herbs, which exerts antioxidative, antiinflammatory, antiallergic, and analgesic effects [46]. Recent studies have shown that quercetin can reduce the growth and invasion of tumor cells [47, 48]. Quercetin has anti-inflammatory activity by inhibiting the production of cyclooxygenase (COX) and lipoxygenase (LOX), which are considered to be closely related to inflammation [49, 50]. In addition, this compound shows remarkable antinociceptive and neuroprotective effects in animal models and inhibits light edema formation [51, 52]. Another molecule identified was catechin, which has anti-inflammatory and antioxidant activities and can reduce abnormal sensation after chemotherapy [53, 54]. Other active compounds identified, such as formononetin [55], isorhamnetin [56], and kaempferol [57], have been shown to be effective in inhibiting inflammation and analgesia. It is worth noting that we found that the targets of HQGZWWD can be mapped to the endocrine system and to respiratory system diseases, which are especially enriched in cancer. In recent years, TCM has attracted much attention in improving cancer and its side effects, which is consistent with our results suggesting that HQGZWWD exerts pharmacological effect on many diseases, including CIPN, through the synergistic action of many compounds and different targets. This may define this herbal formula as having multitarget and multifunction characteristics.

Finally, we targeted HQGZWWD and CIPN, as a key approach in excavating the core treatment mechanism of the TCM. We found that TP53, ALB, IL6, VEGFA, CASP3, TNF, and CYP3A4 may play core regulatory roles in treatment. Among these, TP53 is a highly tumor-related gene. Mutant TP53 promotes the proliferation, migration, survival, and invasion of tumor cells, enhances drug resistance, and promotes the metabolism of tumor cells [58]. Both CASP3 and CASP4 belong to the caspase family, which can maintain homeostasis by regulating apoptosis and inflammation $[59,60]$. VEGFA can protect neurons by promoting neovascularization and vascular permeability. Vencappa et al. [61] demonstrated that VEGFA could prevent cisplatin-induced sensory neuronal damage. In 


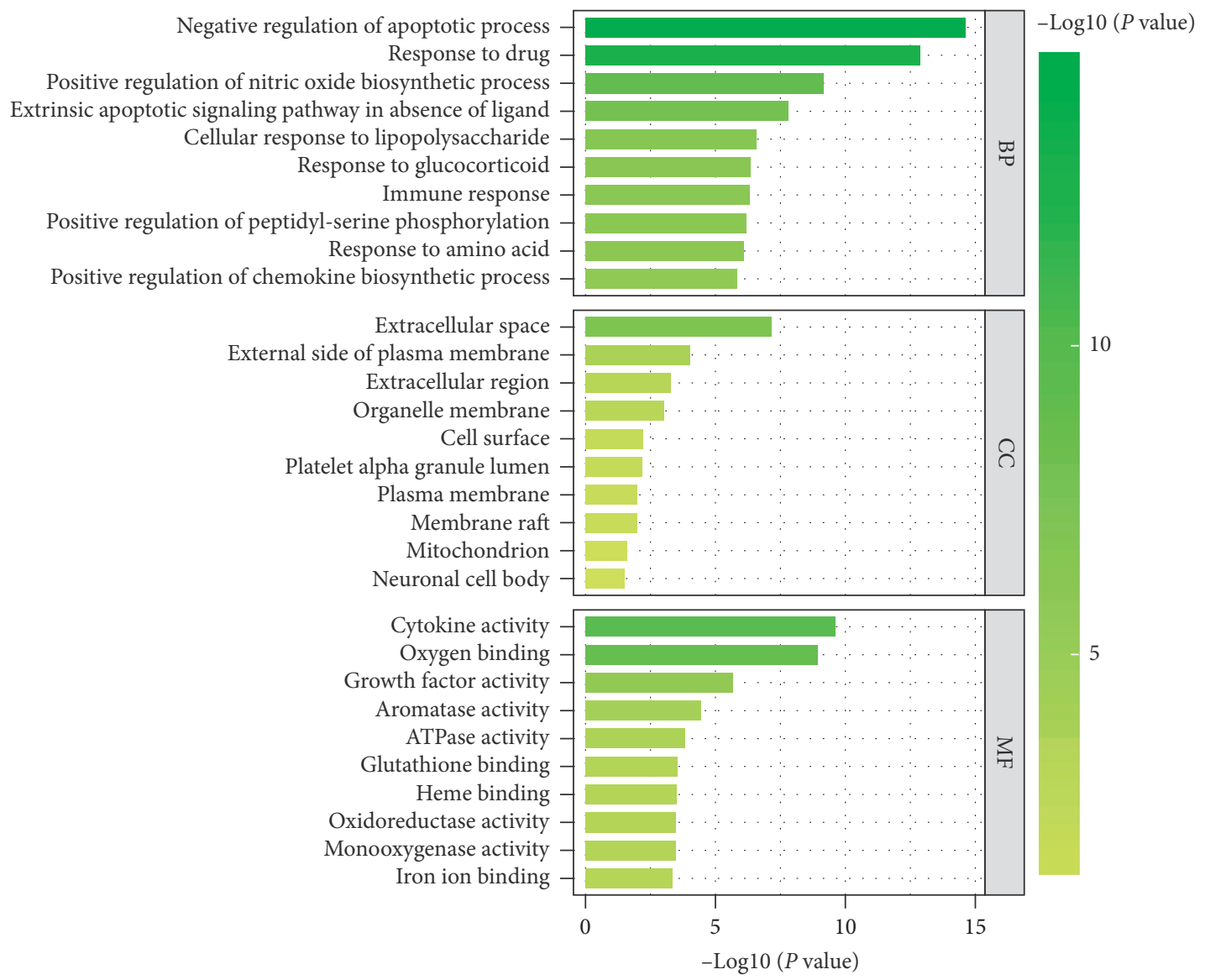

(a)

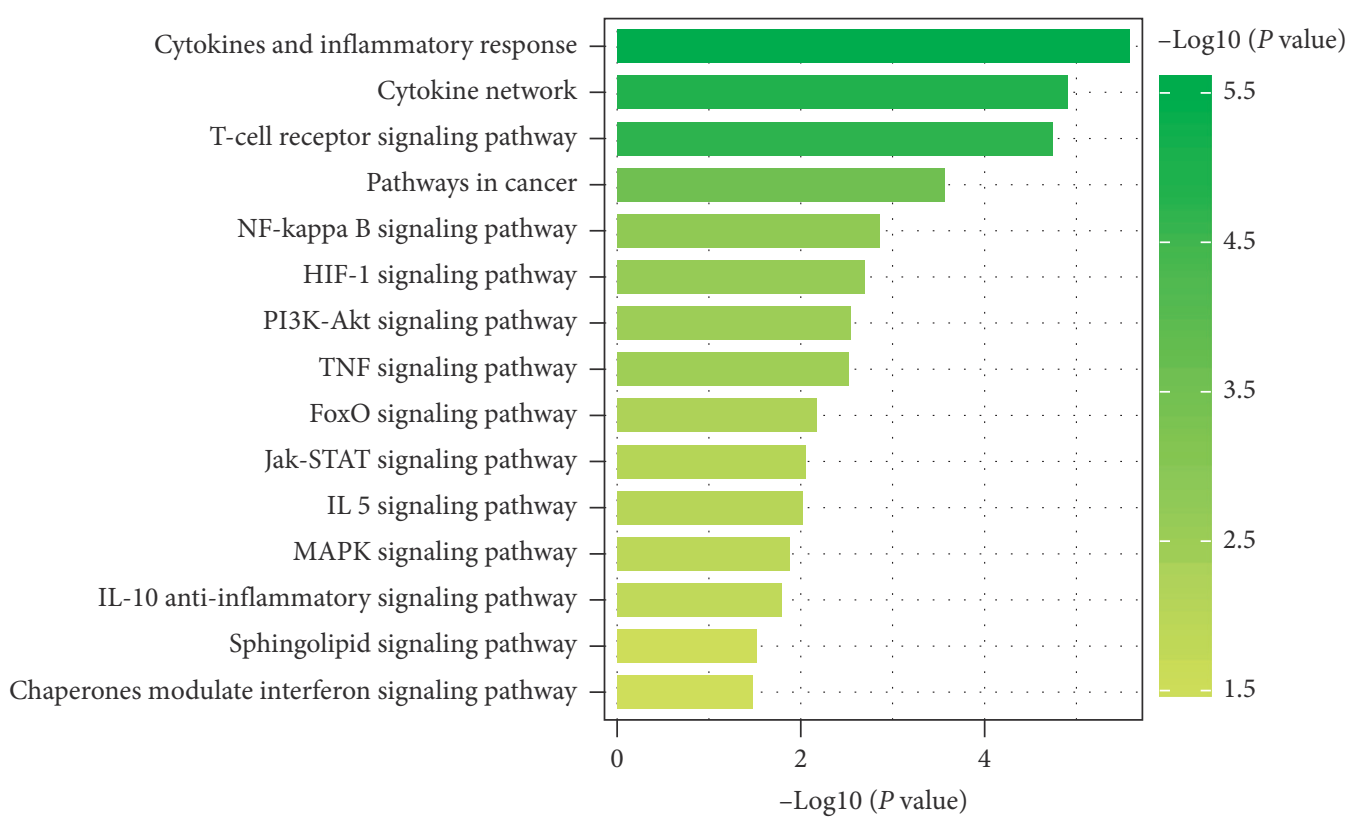

(b)

FIGURE 7: (a) GO functional analysis of overlapping targets. (b) KEGG pathway enrichment analysis of overlapping targets.

addition, inflammation-related targets such as IL6 and TNF are also core targets of CIPN. The GO function analysis of overlapping targets showed that the main biological processes of these targets were the positive regulation of nitric oxide biosynthesis, immune responses, and responses to glucocorticoids, which are similar to CIPN. While KEGG pathway enrichment 
analysis showed that the targets that were mainly enriched included cytokines and inflammatory response, elements of the cytokine network, and the T-cell receptor, NF-kappa B, HIF-1, and PI3K-Akt signaling pathways, which were mostly associated with the inflammatory response. For example, the HIF-1 signaling pathway participates in many biological processes such as hypoxia adaptation, inflammatory development, and tumor growth and plays a key role in inflammation and mediates tumorigenesis $[62,63]$. We found that these pathways intersect with the disease pathway of CIPN, indicating that HQGZWWD has a certain pertinence in the treatment of CIPN.

Overall, our results show that HQGZWWD plays a therapeutic role in CIPN by regulating the inflammatory response and repairing nerve injury, thus providing support for the reliable efficacy of this herbal formula. In addition, in the overlapping targets network, we identified two potential targets (CDK7 and GSTM2) from the prediction platform, which have not been verified by experiments, and represent a clear direction for future research as novel targets for the treatment of CIPN.

Our innovation in this study lies in our integration of multiple TCM databases, which may compensate for the deficiency of incomplete data. Further, our supplementary analysis of the targets for CIPN treatment through a prediction model provides new information for the future study of pathological processes and therapeutic targets. Finally, this study fully illustrates that TCM possesses the characteristic of an active multicompound, multitarget, and multipathway formula, which is of great significance in the study of the curative effect of herbal formulations. However, there are some issues that cannot be overlooked; although the selection of effective compounds is well founded (mainly recommended by databases), they are mostly reported in a single plant, which does not mean that they are still present and play a therapeutic role in the traditional preparation. As mentioned above, we found only about $30 \%$ of the effective compounds in a mass spectrometric analysis of HQGZWWD. Therefore, more mass spectrometric analysis needs to be carried out to determine the effective compounds of traditional preparations.

\section{Conclusion}

Through network pharmacology, we found that HQGZWWD has a significant advantage in the treatment of CIPN. At the same time, the underlying molecular biological mechanisms have been revealed by analyzing the potential core targets, biological functions, and signal pathways involved. Our study provides a theoretical basis for the clinical application of HQGZWWD for the treatment of CIPN.

\section{Data Availability}

The data used to support the findings of this study are available from the corresponding author upon request.

\section{Conflicts of Interest}

The authors declare that there are no conflicts of interest regarding the publication of this paper.

\section{Authors' Contributions}

Jialin Gu and Guoli Wei contributed equally to this work.

\section{Supplementary Materials}

Supplementary 1. Table S1: compounds identified in the HQGZWWD formula. Supplementary 2. Table S2: effective compounds identified in the HQGZWWD formula. Supplementary 3. Table S3: targets of the HQGZWWD formula. Supplementary 4. Table S4: targets of CIPN. (Supplementary Materials)

\section{Acknowledgments}

This study was supported by grants from the Project of Provincial TCM Leading Talent of Jiangsu (no. SLJ0211) and the Project of National Clinical Research Base of TCM in Jiangsu Province (no. JD2019SZXYB04).

\section{References}

[1] L. A. Colvin, "Chemotherapy-induced peripheral neuropathy: where are we now?" Pain, vol. 160, no. Suppl 1, pp. S1-S10, 2019.

[2] J. Ma, A. Kavelaars, P. M. Dougherty, and C. J. Heijnen, "Beyond symptomatic relief for chemotherapy-induced peripheral neuropathy: targeting the source," Cancer, vol. 124, no. 11, pp. 2289-2298, 2018.

[3] P. Zis, A. Paladini, A. Piroli, P. C. McHugh, G. Varrassi, and M. Hadjivassiliou, "Pain as a first manifestation of paraneoplastic neuropathies: a systematic review and metaanalysis," Pain and Therapy, vol. 6, no. 2, pp. 143-151, 2017.

[4] V. Brozou, A. Vadalouca, and P. Zis, "Pain in platin-induced neuropathies: a systematic review and meta-analysis," Pain and Therapy, vol. 7, no. 1, pp. 105-119, 2018.

[5] R. Avan, G. Janbabaei, N. Hendouei et al., "The effect of pregabalin and duloxetine treatment on quality of life of breast cancer patients with taxane-induced sensory neuropathy: a randomized clinical trial," Journal of Research in Medical Sciences, vol. 23, p. 52, 2018.

[6] L. Ye, Y. Jia, K. Ji et al., "Traditional Chinese medicine in the prevention and treatment of cancer and cancer metastasis," Oncology Letters, vol. 10, no. 3, pp. 1240-1250, 2015.

[7] J. Nie, C. Zhao, L. Deng et al., "Efficacy of traditional Chinese medicine in treating cancer," Biomedical Reports, vol. 4, no. 1, pp. 3-14, 2016.

[8] F. Qi, L. Zhao, A. Zhou et al., "The advantages of using traditional Chinese medicine as an adjunctive therapy in the whole course of cancer treatment instead of only terminal stage of cancer," Bioscience Trends, vol. 9, no. 1, pp. 16-34, 2015.

[9] X. Cheng, J. G. Huo, D. Wang et al., "Herbal medicine AC591 prevents oxaliplatin-induced peripheral neuropathy in animal model and cancer patients," Frontiers in Pharmacology, vol. 8, no. 11, p. 344, 2017.

[10] A. L. Hopkins, "Network pharmacology: the next paradigm in drug discovery," Nature Chemical Biology, vol. 4, no. 11, pp. 682-690, 2008.

[11] H. Y. Fang, H. W. Zeng, L. M. Lin et al., "A network-based method for mechanistic investigation of Shexiang Baoxin Pill's treatment of cardiovascular diseases," Scientific Reports, vol. 7, no. 1, Article ID 43632, 2017. 
[12] J. Fang, L. Wang, T. Wu et al., "Network pharmacology-based study on the mechanism of action for herbal medicines in Alzheimer treatment," Journal of Ethnopharmacology, vol. 196, pp. 281-292, 2017.

[13] Q. Ge, L. Chen, M. Tang et al., “Analysis of mulberry leaf components in the treatment of diabetes using network pharmacology," European Journal of Pharmacology, vol. 833, pp. 50-62, 2018.

[14] L. Zeng and K. Yang, "Exploring the pharmacological mechanism of Yanghe decoction on HER2-positive breast cancer by a network pharmacology approach," Journal of Ethnopharmacology, vol. 199, pp. 68-85, 2017.

[15] G. Yu, Y. Zhang, W. Ren et al., "Network pharmacology-based identification of key pharmacological pathways of YinHuang-Qing-Fei capsule acting on chronic bronchitis," International Journal of COPD, vol. 12, pp. 85-94, 2017.

[16] N. Wang, G. Zhao, Y. Zhang et al., "A network pharmacology approach to determine the active components and potential targets of Curculigo Orchioides in the treatment of osteoporosis," Medical Science Monitor, vol. 23, pp. 5113-5122, 2017.

[17] P. J. Eddershaw, A. P. Beresford, and M. K. Bayliss, “ADME/ PK as part of a rational approach to drug discovery," Drug Discovery Today, vol. 5, no. 9, pp. 409-414, 2000.

[18] J. Ru, P. Li, J. Wang et al., "TCMSP: a database of systems pharmacology for drug discovery from herbal medicines," Journal of Cheminformatics, vol. 6, no. 13, 2014.

[19] X. Xu, W. Zhang, C. Huang et al., "A novel chemometric method for the prediction of human oral bioavailability," International Journal of Molecular Sciences, vol. 13, no. 6, pp. 6964-6982, 2012.

[20] W. Tao, X. Xu, X. Wang et al., "Network pharmacology-based prediction of the active ingredients and potential targets of Chinese herbal Radix Curcumae formula for application to cardiovascular disease," Journal of Ethnopharmacology, vol. 145, no. 1, pp. 1-10, 2013.

[21] H.-Y. Xu, Y.-Q. Zhang, Z.-M. Liu et al., "ETCM: an encyclopaedia of traditional Chinese medicine," Nucleic Acids Research, vol. 47, no. D1, pp. D976-D982, 2019.

[22] X. Liu, S. Ouyang, B. Yu et al., "PharmMapper server: a web server for potential drug target identification using pharmacophore mapping approach," Nucleic Acids Research, vol. 38, no. suppl_2, pp. W609-W614, 2010.

[23] Z.-J. Yao, J. Dong, Y.-J. Che et al., "TargetNet: a web service for predicting potential drug-target interaction profiling via multi-target SAR models," Journal of Computer-Aided Molecular Design, vol. 30, no. 5, pp. 413-424, 2016.

[24] M. Safran, I. Dalah, J. Alexander et al., "GeneCards Version 3: the human gene integrator," Database (Oxford), vol. 2010, baq020 pages, 2010 .

[25] J. Piñero, J. M. Ramírez-Anguita, J. Saüch-Pitarch et al., "The DisGeNET knowledge platform for disease genomics: 2019 update," Nucleic Acids Research, vol. 48, no. D1, pp. D845-D855, 2020.

[26] M. Franz, H. Rodriguez, C. Lopes et al., "GeneMANIA update 2018,” Nucleic Acids Research, vol. 46, pp. W60-W64, 2018.

[27] D. Szklarczyk, A. L. Gable, D. Lyon et al., "STRING v11: protein-protein association networks with increased coverage, supporting functional discovery in genome-wide experimental datasets," Nucleic Acids Research, vol. 47, no. D1, pp. D607-D613, 2019.

[28] P. Shannon, A. Markiel, O. Ozier et al., "Cytoscape: a software environment for integrated models of biomolecular interaction networks," Genome Research, vol. 13, no. 11, pp. 2498-2504, 2003.

[29] D. W. Huang, B. T. Sherman, and R. A. Lempicki, "Systematic and integrative analysis of large gene lists using DAVID bioinformatics resources," Nature Protocols, vol. 4, no. 1, pp. 44-57, 2009.

[30] V. A. Carozzi, A. Canta, and A. Chiorazzi, "Chemotherapyinduced peripheral neuropathy: what do we know about mechanisms?" Neuroscience Letters, vol. 596, pp. 90-107, 2015.

[31] T. J. Kaley and L. M. Deangelis, "Therapy of chemotherapyinduced peripheral neuropathy," British Journal of Haematology, vol. 145, no. 1, pp. 3-14, 2009.

[32] D. R. Pachman, D. L. Barton, J. C. Watson, and C. L. Loprinzi, "Chemotherapy-induced peripheral neuropathy: prevention and treatment," Clinical Pharmacology \& Therapeutics, vol. 90, no. 3, pp. 377-387, 2011.

[33] S. Hou, B. Huh, H. K. Kim, K. H Kim, and S Abdi, "Treatment of chemotherapy-induced peripheral neuropathy: systematic review and recommendations," Pain Physician, vol. 21, no. 6, pp. 571-592, 2018.

[34] E. M. L. Smith, H. Pang, C. Cirrincione et al., "Effect of duloxetine on pain, function, and quality of life among patients with chemotherapy-induced painful peripheral neuropathy," JAMA, vol. 309, no. 13, pp. 1359-1367, 2013.

[35] M. Takenaka, H. Iida, S. Matsumoto, S. Yamaguchi, N. Yoshimura, and M. Miyamoto, "Successful treatment by adding duloxetine to pregabalin for peripheral neuropathy induced by paclitaxel," American Journal of Hospice and Palliative Medicine, vol. 30, no. 7, pp. 734-736, 2013.

[36] C. Brami, T. Bao, and G. Deng, "Natural products and complementary therapies for chemotherapy-induced peripheral neuropathy: a systematic review," Critical Reviews in Oncology/hematology, vol. 98, pp. 325-334, 2016.

[37] G. L. Wei, Z. C. Gu, L. C. Li et al., "Experimental study of Huangqi Guizhi Wuwu decoction to prevent oxaliplatin-induced chronic peripheral neuropathy by reducing platinum accumulation," Modernization of Traditional Chinese Medicine and Materia Medica-World Science and Technology, vol. 21, no. 07, pp. 1467-1473, 2019.

[38] J. Tian, Z. Q. Yao, X. Y. Wu et al., "Systematic review and meta-analysis on efficacy of Huangqi Guizhi Wuwu decoction for oxaliplatin-induced peripheral neurotoxicity," Chinese Journal of Experimental Traditional Medical Formulae, vol. 19, no. 22, pp. 325-330, 2013.

[39] B. B. Aggarwal, A. B. Kunnumakkara, K. B. Harikumar et al., "Signal transducer and activator of transcription-3, inflammation, and cancer," Annals of the New York Academy of Sciences, vol. 1171, no. 1, pp. 59-76, 2009.

[40] D. Masciocchi, A. Gelain, S. Villa, F. Meneghetti, and D. Barlocco, "Signal transducer and activator of transcription 3 (STAT3): a promising target for anticancer therapy," Future Medicinal Chemistry, vol. 3, no. 5, pp. 567-597, 2011.

[41] S. Dziennis and N. J. Alkayed, "Role of signal transducer and activator of transcription 3 in neuronal survival and regeneration," Reviews in the Neurosciences, vol. 19, no. 4-5, pp. 341-361, 2008.

[42] Y. Q. Zhou, Z. Liu, Z. H. Liu et al., "Interleukin-6: an emerging regulator of pathological pain," Journal of Neuroinflammation, vol. 13, no. 1, p. 141, 2016.

[43] X.-M. Wang, T. J. Lehky, J. M. Brell, and S. G. Dorsey, "Discovering cytokines as targets for chemotherapy-induced painful peripheral neuropathy," Cytokine, vol. 59, no. 1, pp. 3-9, 2012. 
[44] D. W. Zochodne and D. Levy, "Nitric oxide in damage, disease and repair of the peripheral nervous system," Cellular and Molecular Biology (Noisy-Le-Grand, France), vol. 51, no. 3, pp. 255-267, 2005.

[45] E. Dominguez, C. Rivat, B. Pommier, A. Mauborgne, and M. Pohl, "JAK/STAT3 pathway is activated in spinal cord microglia after peripheral nerve injury and contributes to neuropathic pain development in rat," Journal of Neurochemistry, vol. 107, no. 1, pp. 50-60, 2008.

[46] S. Kundur, A. Prayag, P. Selvakumar et al., "Synergistic anticancer action of quercetin and curcumin against triple-negative breast cancer cell lines," Journal of Cellular Physiology, vol. 234, no. 7, pp. 1103-1118, 2019.

[47] S. Sun, F. Gong, P. Liu, and Q. Miao, "Metformin combined with quercetin synergistically repressed prostate cancer cells via inhibition of VEGF/PI3K/Akt signaling pathway," Gene, vol. 664, pp. 50-57, 2018.

[48] Y.-H. Lee and P.-T. Tuyet, "Synthesis and biological evaluation of quercetin-zinc (II) complex for anti-cancer and antimetastasis of human bladder cancer cells," In Vitro Cellular \& Developmental Biology - Animal, vol. 55, no. 6, pp. 395-404, 2019.

[49] H. P. Kim, I. Mani, L. Iversen, and V. A. Ziboh, "Effects of naturally-occurring flavonoids and biflavonoids on epidermal cyclooxygenase and lipoxygenase from Guinea-pigs," Prostaglandins, Leukotrienes and Essential Fatty Acids, vol. 58, no. 1, pp. 17-24, 1998.

[50] K. M. Lee, M. K. Hwang, D. E. Lee, K. W. Lee, and H. J. Lee, "Protective effect of quercetin against arsenite-induced COX2 expression by targeting PI3K in rat liver epithelial cells," Journal of Agricultural and Food Chemistry, vol. 58, no. 9, pp. 5815-5820, 2010.

[51] K. S. Raygude, A. D. Kandhare, P. Ghosh, A. E. Ghule, and S. L. Bodhankar, "Evaluation of ameliorative effect of quercetin in experimental model of alcoholic neuropathy in rats," Inflammopharmacology, vol. 20, no. 6, pp. 331-341, 2012.

[52] M. I. Azevedo, A. F. Pereira, R. B. Nogueira et al., "The antioxidant effects of the flavonoids rutin and quercetin inhibit oxaliplatin-induced chronic painful peripheral neuropathy," Molecular Pain, vol. 9, p. 53, 2013.

[53] J. S. Lee, Y. T. Kim, E. K. Jeon et al., "Effect of green tea extracts on oxaliplatin-induced peripheral neuropathy in rats," BMC Complementary and Alternative Medicine, vol. 12, p. 124, 2012.

[54] N. T. Zaveri, "Green tea and its polyphenolic catechins: medicinal uses in cancer and noncancer applications," Life Sciences, vol. 78, no. 18, pp. 2073-2080, 2006.

[55] R. Lima Cavendish, J. de Souza Santos, R. Belo Neto et al., "Antinociceptive and anti-inflammatory effects of Brazilian red propolis extract and formononetin in rodents," Journal of Ethnopharmacology, vol. 173, pp. 127-133, 2015.

[56] K. Seo, J. H. Yang, S. C. Kim et al., "The antioxidant effects of isorhamnetin contribute to inhibit COX-2 expression in response to inflammation: a potential role of HO-1," Inflammation, vol. 37, no. 3, pp. 712-722, 2014.

[57] P. Rajendran, T. Rengarajan, N. Nandakumar et al., "Kaempferol, a potential cytostatic and cure for inflammatory disorders," European Journal of Medicinal Chemistry, vol. 86, pp. 103-112, 2014.

[58] R. S. Warren, C. E. Atreya, D. Niedzwiecki et al., "Association of TP53 mutational status and gender with survival after adjuvant treatment for stage III colon cancer: results of CALGB 89803," Clinical Cancer Research, vol. 19, no. 20, pp. 5777-5787, 2013.
[59] Z.-J. Dai, J. Gao, Z.-Z. Ji et al., "Matrine induces apoptosis in gastric carcinoma cells via alteration of Fas/FasL and activation of caspase-3," Journal of Ethnopharmacology, vol. 123, no. 1, pp. 91-96, 2009.

[60] G. Sollberger, G. E. Strittmatter, M. Kistowska et al., "Caspase4 is required for activation of inflammasomes," Journal of Immunology, vol. 188, no. 4, pp. 1992-2000, 2012.

[61] S. Vencappa, L. F. Donaldson, and R. P. Hulse, "Cisplatin induced sensory neuropathy is prevented by vascular endothelial growth factor-A," American Journal of Translational Research, vol. 7, no. 6, pp. 1032-1044, 2015.

[62] N. Dehne and B. Brüne, "HIF-1 in the inflammatory microenvironment," Experimental Cell Research, vol. 315, no. 11, pp. 1791-1797, 2009.

[63] K. Balamurugan, "HIF-1 at the crossroads of hypoxia, inflammation, and cancer," International Journal of Cancer, vol. 138, no. 5, pp. 1058-1066, 2016. 